\title{
Is Bifidobacterium breve effective in the treatment of childhood constipation? Results from a pilot study
}

\author{
MM Tabbers, I de Milliano, MG Roseboom, MA Benninga
}

\begin{abstract}
Background: Probiotics are increasingly used in the treatment of functional gastrointestinal disorders. Studies in constipated adults with a Bifidus yoghurt (containing Bifidobacterium breve, Bifidobacterium bifidum and Lactobacillus acidophilus) showed a significant increase in defecation frequency. The aim of this pilot study was to determine if Bifidobacterium breve is effective in the treatment of childhood constipation.

Methods: Children, 3 to 16 years of age, with functional constipation according to the Rome III criteria were eligible for this study. During 4 weeks, children received one sachet of powder daily, containing $10^{8}-10^{10} \mathrm{CFU}$ Bifidobacterium breve. Furthermore, children were instructed to try to defecate on the toilet for 5-10 minutes after each meal and to complete a standardized bowel diary daily. The primary outcome measure was change in defecation frequency. Secondary outcome measures were stool consistency using the Bristol stool scale frequency of episodes of faecal incontinence, pain during defecation, frequency of abdominal pain, frequency of adverse effects (nausea, diarrhoea and bad taste), and frequency of intake of bisacodyl.

Results: Twenty children (75\% male, mean age 7.4) were included in this pilot study. The defecation frequency per week significantly increased from $0.9(0-2)$ at baseline to $4.9(0-21)$ in week $4(p<0.01)$. The mean stool consistency score increased from $2.6(2-4)$ at baseline to $3.5(1-6)$ in week $4(p=0.03)$. The number of faecal incontinence episodes per week significantly decreased from $9.0(0-35)$ at baseline to $1.5(0-7)$ in week $4(p<0.01)$. Abdominal pain episodes per week significantly decreased from $4.2(0-7)$ at baseline to $1.9(0-7)$ in week $4(p=$ 0.01). No side effects occurred.
\end{abstract}

Conclusion: Bifidobacterium breve is effective in increasing stool frequency in children with functional constipation. Furthermore it has a positive effect with respect to stool consistency, decreasing the number of faecal incontinence episodes and in diminishing abdominal pain. A randomized placebo controlled trial is required to confirm these data.

\section{Background}

Functional constipation is a common and frustrating problem in childhood with an estimated prevalence of $3 \%$ in the western world [1]. This chronic condition is characterised by infrequent defecation less than three times per week, more than two episodes of faecal incontinence per week, the passage of large and painful stools which clog the toilet and retentive posturing. Upon physical examination a palpable faecal mass is often found

\footnotetext{
* Correspondence: m.m.tabbers@amc.nl

Department of Paediatric Gastroenterology and Nutrition, Emma Children's Hospital/Academic Medical Centre, Amsterdam, The Netherlands
}

in the abdomen and the rectum [2,3]. It causes distress to child and family and results in severe emotional disturbance and family discord [4]. The pathophysiology underlying functional constipation is undoubtedly multifactorial, and not well understood. Withholding behaviour is probably the major cause for the development of constipation and might be caused by the previous production of a large, hard painful stool, anal fissures, a primarily behavioural mechanism or the resistance to go to another toilet then their own [4].

To date, patients are treated with a combination of education, toilet training and oral laxatives. Disappointingly, only $50 \%$ of all children followed for 6 to 
12 months are found to recover and were successfully taken off laxatives [5]. Another study showed that despite intensive medical and behavioural therapy, 25\% of patients developing constipation before the age of 5 years continued to have severe complaints of constipation beyond puberty [6]. Furthermore, in 50\% of the patients using these compounds, adverse side-effects were registered such as: abdominal pain, bloating, flatulence, diarrhoea, nausea and bad taste [7]. No data exist concerning possible long-term adverse effects such as electrolyte disturbances, mucosal damage and habituation.

Probiotics are defined as live micro-organisms which when administered in adequate amounts, $10^{6}-10^{9}$ colony forming units, confer a health benefit on the host [8]. The use of probiotics has entered mainstream medicine and has proven being an effective therapy in many different gastrointestinal disorders, including functional gastrointestinal disorders $[9,10]$. However, large trials investigating the efficacy and safety of probiotics in pediatric patients are lacking $[11,12]$. Studies in constipated adults with a Bifidus yoghurt, containing Bifidobacterium breve, Bifidobacterium bifidum and Lactobacillus acidophilus, showed a significant increase in defecation frequency without any side effects [13,14]. Furthermore, a randomized controlled trial using Bifidobacterium breve in preterm infants showed less gas accumulation in the stomach, less vomiting and improved weight gain without any side effects, suggesting a positive effect on gastrointestinal motility [15] The exact working mechanism of probiotics are not well understood. There are some hypotheses, however, why probiotics might have therapeutic potential for the treatment of constipation. Firstly, a dysbiosis in the gut flora in constipated patients has been suggested which might improve after the ingestion of probiotics $[9,10]$. It remains important however to understand if dysbiosis is a secondary manifestation of constipation, or if it is a factor contributing to constipation. Furthermore, probiotics can lower $\mathrm{pH}$ of the colon by producing lactic, acetic and other short chain fatty acids. A lower $\mathrm{pH}$ enhances colonic peristalsis and subsequently decreases colonic transit time $[9,10]$.

Based on the positive data in constipated adults, we therefore performed a pilot study to determine if Bifidobacterium breve is effective in the treatment of children with constipation.

\section{Methods}

\section{Subjects}

Children, 3 to 16 years of age, referred to the outpatient clinic of a tertiary pediatric gastroenterology department, with constipation were eligible for this study. Patients were included if they had been suffering from functional constipation according to the Rome III criteria for the last 2 months [2,3]. All children included had a defecation frequency of $<3$ times/week and one or more of the following criteria: faecal incontinence $>1$ episode/week, a large amount of stools that clog the toilet, painful defecation, withholding behaviour, or abdominal or rectal faecal impaction upon physical examination. In order to obtain a homogeneous group of patients, we included children with a defecation frequency $<3$ times/ week in combination with at least one other ROME III criterion. Patients were not enrolled in this study if they had been treated for constipation less than 2 weeks before the start of the study. Other exclusion criteria were: a diagnosis of either IBS or functional nonretentive faecal incontinence according to the Rome III criteria; a diagnosis of mental retardation or metabolic disease (hypothyroidism), Hirschsprung's disease, spinal anomalies, anorectal pathology, previous gastrointestinal surgery. All children older than 12 years and/or parents gave informed consent. This pilot was approved by the medical ethical committee,

\section{Study design}

Seven days prior to baseline assessment and during the treatment period, all children recorded frequency of bowel movements, stool consistency according to the Bristol stool scale, the number of faecal incontinence episodes, pain during defecation, abdominal pain, as well as adverse effects such as vomiting and diarrhoea in a standardized bowel diary. At baseline a medical history and information on the current defection pattern was collected and also a physical examination including a rectal digital exam was performed. Information and education about functional constipation was given to all patients and their care takers. Before start of the probiotic treatment, all children received once daily for 3 days a rectal enema in order to accomplish rectal disimpaction. After rectal disimpaction, all children received daily one sachet of powder containing $10^{8-10}$ CFU Bifidobacterium breve Yakult, for 4 weeks. The patients were allowed to mix the powder with all liquids on condition that the liquid was not hot. During the study, all children were instructed to try to defecate on the toilet for 5-10 minutes after each meal (3 times a day). Patients were not allowed to consume any other fermented dairy product during this study or any other laxatives, except for the rescue medication Biscaodyl. Names of the forbidden products were pointed out in the diary. During the product consumption period, patients were instructed to take bisacodyl $5 \mathrm{mg}$ if they did not defecate for 3 consecutive days. Clinical evaluation, frequency of adverse effects and compliance to the study protocol were carried out at enrolment and at 2, 4 and 6 weeks using standardized bowel diaries. 


\section{Outcome measures}

The primary outcome measure was change in defecation frequency in week 4 compared to baseline. Secondary outcome measures were stool consistency, frequency of episodes of faecal incontinence, pain during defecation, frequency of abdominal pain, frequency of adverse effects (nausea, diarrhoea and bad taste), and frequency of intake of bisacodyl.

\section{Analysis}

Descriptive statistics were performed for baseline characteristics, adverse effects and Bisacodyl use. Change of frequency of bowel movements, fecal incontinence and change of stool consistency, was assessed using the nonparametric paired Wilcoxon test. For the comparison of abdominal pain between baseline and the evaluation time points, the Wilcoxon rank test was used. A p-value $<0.05$ was considered to be significant. All analyses were done with SPSS (version 16.0).

\section{Results}

Between July 2009 and January 2010, 22 children were included into this pilot study. Two children were lost to follow-up without having any outcome data during follow-up. Both patients were therefore excluded from the final analysis. All outcomes of the remaining 20 children were collected and analyzed. The baseline characteristics are described in Table 1 (mean (range))

The defecation frequency per week significantly increased from $0.9(0-2)$ at baseline to $5.3(0-21)$ in week $2(\mathrm{p}<0.01)$ and $4.9(0-21)$ in week $4(\mathrm{p}<0.01)$ (figure 1 : Defecation frequency over 4 weeks.). The mean stool consistency score significantly increased from 2.6 (2-4) at baseline to $3.6(1-6)$ in week $2(p=0.07)$ and $3.5(1-6)$ in week $4(\mathrm{p}=0.03)$. The episodes of faecal incontinence per week significantly decreased from $9.0(0-35)$ at baseline to $2.6(0-7)$ in week $2(\mathrm{p}=0.01)$ and $1.5(0-7)$ in week 4 ( $\mathrm{p}<0.01)$. Pain during defecation decreased from $71 \%(12 / 17)$ at baseline to $40 \%(8 / 20)$ in week 2 (p = $0.10)$ and $33 \%(6 / 18)$ in week $4(\mathrm{p}=0.08)$. Abdominal pain episodes per week significantly decreased from 4.2 $(0-7)$ at baseline to $2.2(0-7)$ in week $2(\mathrm{p}=0.02)$ and 1.9 $(0-7)$ in week $4(\mathrm{p}=0.01)$. Bisacodyl was used by $45 \%$ of patients during week $1,25 \%$ of patients during week 2 ,

\section{Table 1 Baseline patient characteristics: mean (range)}

\begin{tabular}{ll}
\hline Age in years & $7.4(4-13)$ \\
Boys, n (\%) & $15(75 \%)$ \\
Mean stool frequency per week & $0.9(0-2)$ \\
Stool consistency score & $2.6(2-4)$ \\
Faecal incontinence episodes per week & $9(0-35)$ \\
Pain during defecation & $71 \%(12 / 17)$ \\
Abdominal pain episodes per week & $4.2(0-7)$ \\
\hline
\end{tabular}

$35 \%$ of patients during week 3 and by $20 \%$ of patients during week 4 . No side effects were reported such as nausea, diarrhoea and bad taste or increased flatulence during the study period. Table 2 gives an overview of the outcome measures with p-values.

\section{Discussion}

This pilot study showed that intake of Bifidobacterium breve for 4 weeks, significantly increased the defecation frequency. Furthermore, stool consistency, the frequency of episodes of faecal incontinence and the frequency of abdominal pain significantly changed after the use of this specific probiotic strain.

A recent systematic review on the effects of laxative treatment and dietary measures in the management of childhood constipation found only 2 randomized controlled trials evaluating the effects of probiotics [16]. In the first small study, 45 children younger than 10 years with chronic constipation were randomly assigned to receive magnesium oxide $\left(50 \mathrm{mg} / \mathrm{kg} /\right.$ day $(\mathrm{n}=18)$, or $8 \times 10^{8} \mathrm{cfu} /$ day of the probiotic Lactobacillus casei rhamnosus ( $\mathrm{n}=$ $18)$, or placebo $(n=9)$ twice daily for 4 weeks [17]. No statistically significant difference in the defecation frequency per day was found between the probiotic group and the magnesium oxide group. However, patients receiving either the probiotic strain or the oral laxative had a significantly higher defecation frequency compared to the placebo group (defecation frequency [times/day $0.57 \pm 0.17$ and $0.55 \pm 0.13$, respectively, compared to $0.37 \pm 0.10, \mathrm{p}=$ 0.03). The second trial was conducted to determine if Lactobacillus rhamnosus GG (LGG) is an effective adjunct to lactulose for treating constipation in children. A total of 48 children with constipation received $1 \mathrm{ml} / \mathrm{kg} /$ day of $70 \%$ lactulose plus $10^{9} \mathrm{cfu}$ of LGG or $1 \mathrm{ml} / \mathrm{kg} /$ day of $70 \%$ lactulose plus placebo, twice daily for 12 weeks [12]. There were no significant differences in rates of product success (defined as $\geq 3$ spontaneous stools per week with no faecal incontinence) at 12 and 24 weeks between the LGG group (rates: $72 \%$ and $64 \%$, respectively) and the placebo group (rates: $68 \%$ and $65 \%$, respectively).

In a recent trial, 44 children, at least 6 months old, with chronic constipation were randomly assigned to receive supplementation with the probiotic Lactobacillus reuteri (DSM 17938) $(\mathrm{n}=22)$ or placebo $(\mathrm{n}=22)$ [18]. Infants receiving Lactobacillus reuteri had a significantly higher frequency of bowel movements than infants receiving a placebo at week 8 of supplementation $(2.82$ per week at week 0 , compared with 4.77 at week 8 in the probiotic group, absolute numbers not given for placebo group, $\mathrm{P}=0.027$ ). There was no significant difference between Lactobacillus reuteri and placebo groups in the stool consistency at all weeks nor in the presence of inconsolable crying episodes. All three trials did not report any adverse events in the probiotic group. 


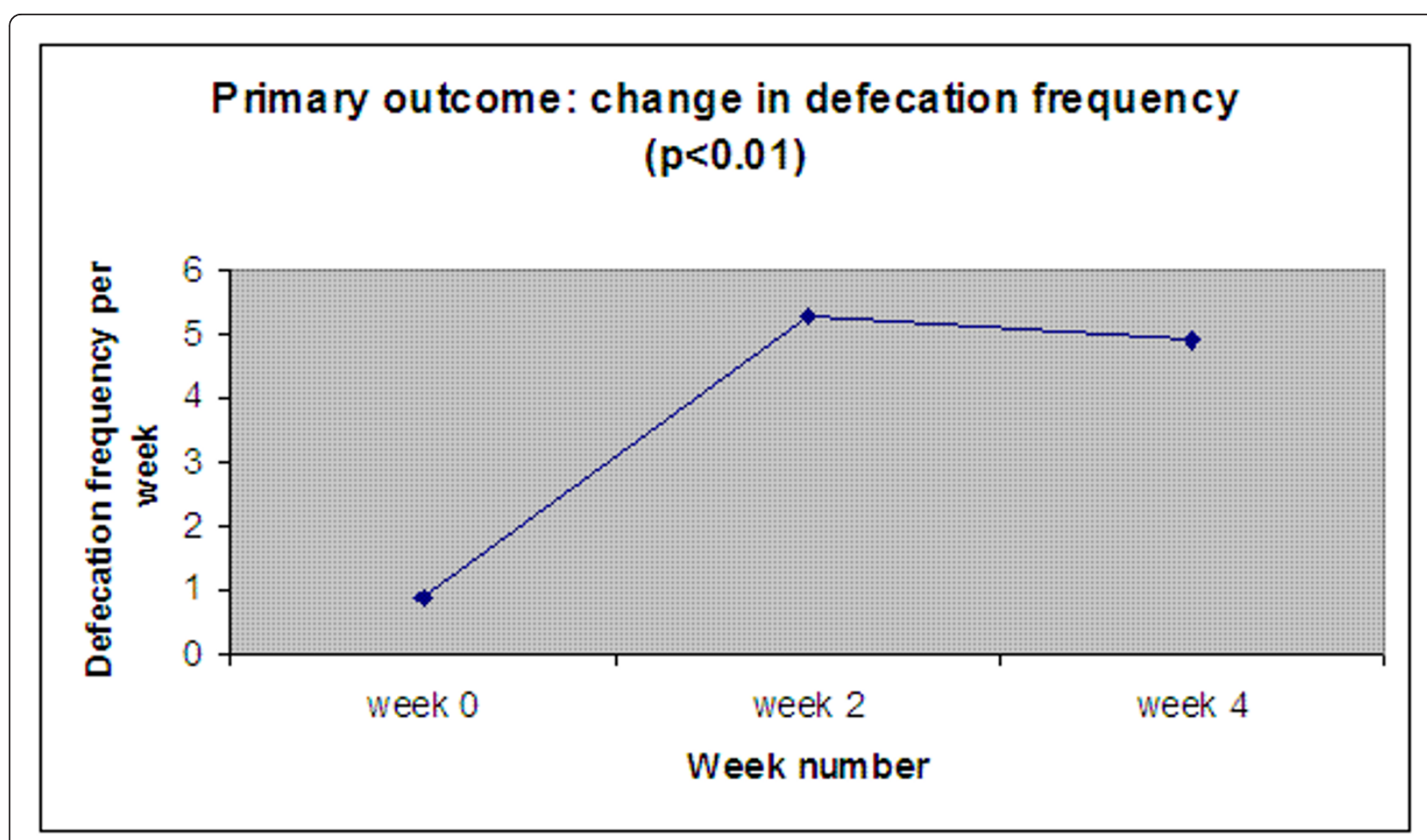

Figure 1 Primary outcome: change in defecation frequency $(\mathbf{n}=\mathbf{2 0})$. X-axis: week number. Y-axis: defecation frequency per week.

A mixture of probiotics (containing Bifidobacteria (B.) bifidum, B. infantis, B. longum, Lactobacillus (L.) casei, L. plantarum and L. rhamnosus), increased the number of bowel movements, decreased the number of faecal incontinence episodes and improved the consistency of stools [19]. Although the results of this small pilot study are positive, a large randomized controlled trial is necessary to confirm these data.

It is unknown why the outcome of the above mentioned trials in constipated children is so different. We speculate that the use of different inclusion criteria for paediatric constipation with consequently different study populations and the use of different probiotic strains, given in different dosages with variable duration of the treatment period, influence study outcomes.

In contrast to the few paediatric studies, data suggest that adults with constipation might benefit from ingestion of B. lactis DN-173 010, L. casei Shirota, and E. coli
Nissle 1917. All studies showed an increased defecation frequency and improved stool consistency [20]. These findings, however are not directly applicable to the paediatric population because constipation in children differs considerably from that in constipated adults with regard to its prevalence, onset, aetiology, symptoms, treatment, and prognosis [21].

Besides the increase in defecation frequency and decrease in episodes of faecal incontinence, this study also showed a significant effect in softening of stools and in decreasing abdominal pain. Both effects could be a direct consequence an increase in defecation but theoretically, it could also be caused by the working mechanism of the probiotics. It has been assumed that probiotics soften the stools by stimulating water and electrolyte secretion [22,23]. Furthermore, one paediatric study and several studies in adults with irritable bowel syndrome (IBS), have demonstrated that abdominal pain

Table 2 Main outcome measures (mean) with p-values $(n=20)$

\begin{tabular}{lllll}
\hline Outcome & At baseline & Week 2 & Week 4 & P-value (week 4-week 0) \\
\hline Defecation frequency p/week & 0.9 & 5.3 & 4.9 & $\mathrm{p}<0.01$ \\
\hline Stool consistency score & 2.6 & 3.6 & 3.5 & $\mathrm{p}=0.03$ \\
\hline Episodes of faecal incontinence p/week & 9.0 & 2.6 & 1.5 & $\mathrm{p}<0.01$ \\
\hline Pain during defecation & $71 \%$ & $40 \%$ & $33 \%$ & $\mathrm{p}=0.08$ \\
\hline Abdominal pain episodes p/week & 4.2 & 2.2 & 1.9 & $\mathrm{p}=0.01$ \\
\hline
\end{tabular}


decreased when using probiotics $[17,24,25]$. Whorwell et al. conducted a randomized trial in 360 women with IBS receiving Bifidobacterium infantis and found a significant improvement of abdominal pain which occurred irrespective of any effect on stool frequency. The authors hypothesized that the probiotics were able to diminish visceral hypersensitivity by its anti-inflammatory effect on the enteric mucosa [26].

According to the available data, it is assumed that the risk of infection with the probiotic lactobacilli or bifidobacteria is similar to risks with commensal strains [10]. However, there is concern that the use of probiotics may result in harmful events in at-risk populations like immunocompromized subjects or in patients with other lifethreatening illnesses, who were admitted in the intensive care unit. Based on our results and their safety profile, adding probiotics to the standard treatment of functional constipation in otherwise healthy children is promising. The major limitation of our study is that this study is a non randomized non placebo controlled small pilot study. However, since this study shows promising results it is worthwhile to perform a large RCT to unravel the efficacy of Bifidobacterium breve in constipated children.

In conclusion, this small pilot study suggests that Bifidobacterium breve is effective in the treatment of childhood constipation. A large randomized placebo controlled trial is now required to confirm these data.

\section{Acknowledgements}

The authors wish to thank the Yakult Central Institute for Microbiological Research, Tokyo, Japan for the supply of product. Yakult Netherlands BV, Amstelveen, The Netherlands has funded this study.

\section{Authors' contributions}

MMT and MAB participated in the design of the study.

All authors collected the data.

MMT and IDM did the statistical analysis.

MMT drafted the first manuscript.

All authors read and approved the final manuscript.

\section{Competing interests}

The authors declare that they have no competing interests.

Received: 13 August 2010 Accepted: 23 February 2011

Published: 23 February 2011

\section{References}

1. van den Berg MM, Benninga MA, Di LC: Epidemiology of childhood constipation: a systematic review. Am J Gastroenterol 2006, 101:2401-2409.

2. Hyman PE, Milla PJ, Benninga MA, Davidson GP, Fleisher DF, Taminiau J: Childhood functional gastrointestinal disorders: neonate/toddler. Gastroenterology 2006, 130:1519-1526.

3. Rasquin A, Di LC, Forbes D, Guiraldes E, Hyams JS, Staiano A, et al: Childhood functional gastrointestinal disorders: child/adolescent. Gastroenterology 2006, 130:1527-1537.

4. Benninga MA, Voskuijl WP, Taminiau JA: Childhood constipation: is there new light in the tunnel? J Pediatr Gastroenterol Nutr 2004, 39:448-464

5. Pijpers MA, Bongers ME, Benninga MA, Berger MY: Functional constipation in children: a systematic review on prognosis and predictive factors. J Pediatr Gastroenterol Nutr 2010, 50:256-268.
6. Bongers ME, van Wijk MP, Reitsma JB, Benninga MA: Long-term prognosis for childhood constipation: clinical outcomes in adulthood. Pediatrics 2010, 126:e156-e162.

7. Voskuijl W, de LF, Verwijs W, Hogeman P, Heijmans J, Makel W, et al: PEG 3350 (Transipeg) versus lactulose in the treatment of childhood functional constipation: a double blind, randomised, controlled, multicentre trial. Gut 2004, 53:1590-1594.

8. Gill HS, Guarner F: Probiotics and human health: a clinical perspective. Postgrad Med J 2004, 80:516-526.

9. Picard C, Fioramonti J, Francois A, Robinson T, Neant F, Matuchansky C: Review article: bifidobacteria as probiotic agents - physiological effects and clinical benefits. Aliment Pharmacol Ther 2005, 22:495-512.

10. Szajewska H, Setty M, Mrukowicz J, Guandalini S: Probiotics in gastrointestinal diseases in children: hard and not-so-hard evidence of efficacy. J Pediatr Gastroenterol Nutr 2006, 42:454-475.

11. Chmielewska A, Szajewska H: Systematic review of randomised controlled trials: probiotics for functional constipation. World I Gastroenterol 2010, 16:69-75.

12. Banaszkiewicz A, Szajewska H: Ineffectiveness of Lactobacillus GG as an adjunct to lactulose for the treatment of constipation in children: a double-blind, placebo-controlled randomized trial. J Pediatr 2005, 146:364-369

13. Tanaka R, Shimosaka K: Investigation of the stool frequency in elderly who are bed ridden and its improvements by ingesting bifidus yogurt. Nippon Ronen Igakkai Zasshi 1982, 19:577-582.

14. Matsui T, lida K, Okumura T, Hatano M: Effect of Bifidus yogurt on the defecation frequency of the elderly. Jpn J Nutrition 2000, 58:213-218.

15. Kitajima H, Sumida Y, Tanaka R, Yuki N, Takayama H, Fujimura M: Early administration of Bifidobacterium breve to preterm infants: randomised controlled trial. Arch Dis Child Fetal Neonatal Ed 1997, 76:F101-F107.

16. Pijpers MA, Tabbers MM, Benninga MA, Berger MY: Currently recommended treatments of childhood constipation are not evidence based: a systematic literature review on the effect of laxative treatment and dietary measures. Arch Dis Child 2009, 94:117-131.

17. Bu LN, Chang MH, Ni YH, Chen HL, Cheng CC: Lactobacillus casei rhamnosus Lcr35 in children with chronic constipation. Pediatr Int 2007, 49:485-490.

18. Coccorullo P, Strisciuglio C, Martinelli M, Miele E, Greco L, Staiano A. Lactobacillus reuteri (DSM 17938) in Infants with Functional Chronic Constipation: A Double-Blind, Randomized, Placebo-Controlled Study. J Pediatr 2010, 157:598-602.

19. Bekkali NL, Bongers ME, van den Berg MM, Liem O, Benninga MA: The role of a probiotics mixture in the treatment of childhood constipation: a pilot study. Nutr J 2007, 6:17.

20. Chmielewska A, Szajewska H: Systematic review of randomised controlled trials: probiotics for functional constipation. World J Gastroenterol 2010, 16:69-75.

21. Solzi G, Di LC: Are constipated children different from constipated adults? Dig Dis 1999, 17:308-315.

22. Moro G, Minoli I, Mosca M, Fanaro S, Jelinek J, Stahl B, et al: Dosage-related bifidogenic effects of galacto- and fructooligosaccharides in formula-fed term infants. J Pediatr Gastroenterol Nutr 2002, 34:291-295.

23. Bongers ME, de LF, Reitsma JB, Groeneweg M, Taminiau JA, Benninga MA: The clinical effect of a new infant formula in term infants with constipation: a double-blind, randomized cross-over trial. Nutr J 2007, 6:8.

24. Niedzielin $\mathrm{K}$, Kordecki H, Birkenfeld B: A controlled, double-blind, randomized study on the efficacy of Lactobacillus plantarum $299 \mathrm{~V}$ in patients with irritable bowel syndrome. Eur J Gastroenterol Hepatol 2001, 13:1143-1147.

25. Whorwell PJ, Altringer L, Morel J, Bond Y, Charbonneau D, O'Mahony L, et al: Efficacy of an encapsulated probiotic Bifidobacterium infantis 35624 in women with irritable bowel syndrome. Am J Gastroenterol 2006, 101:1581-1590.

26. McCarthy J, O'Mahony L, O'Callaghan L, Sheil B, Vaughan EE, Fitzsimons N, et al: Double blind, placebo controlled trial of two probiotic strains in interleukin 10 knockout mice and mechanistic link with cytokine balance. Gut 2003, 52:975-980.

doi:10.1186/1475-2891-10-19

Cite this article as: Tabbers et al: Is Bifidobacterium breve effective in the treatment of childhood constipation? Results from a pilot study. Nutrition Journal 2011 10:19. 